2012

\title{
A conceptual framework for the spatial analysis of landscape genetic data
}

Post-print/Accepted manuscript

Helene H. Wagner

Marie-Josée Fortin

Wagner, H.H. \& Fortin, MJ. Conserv Genet (2013) 14: 253. doi:10.1007/s10592-012-0391-5

The final publication is available at Springer via http://dx.doi.org/10.1007/s10592-012-0391-5

\section{HOW TO CITE TSPACE ITEMS}

Always cite the published version, so the author(s) will receive recognition through services that track citation counts, e.g. Scopus. If you need to cite the page number of the TSpace version (original manuscript or accepted manuscript) because you cannot access the published version, then cite the TSpace version in addition to the published version using the permanent URI (handle) found on the record page. 


\section{A conceptual framework for the spatial analysis of landscape genetic}

2 data

3 Helene H. Wagner ${ }^{1}$ and Marie-Josée Fortin ${ }^{2}$

4

$5 \quad{ }^{1}$ Department of Ecology and Evolutionary Biology, University of Toronto, 3359

6 Mississauga Road, Mississauga, ON, Canada L5L 1C6. Phone: +1-905-569-4702, email address:

7 helene.wagner@utoronto.ca

${ }^{2}$ Department of Ecology and Evolutionary Biology, University of Toronto, 25 Harbord

9 St., Toronto, ON, Canada M5S 3G5. Phone: +1-416-946-7886, email address:

10 mariejosee.fortin@utoronto.ca, 


\section{Abstract}

13 Understanding how landscape heterogeneity constrains gene flow and the spread of adaptive

14 genetic variation is important for biological conservation given current global change. However,

15 the integration of population genetics, landscape ecology and spatial statistics remains an

16 interdisciplinary challenge at the levels of concepts and methods. We present a conceptual

17 framework to relate the spatial distribution of genetic variation to the processes of gene flow and

18 adaptation as regulated by spatial heterogeneity of the environment, while explicitly considering

19 the spatial and temporal dynamics of landscapes, organisms and their genes. When selecting the

20 appropriate analytical methods, it is necessary to consider the effects of multiple processes and

21 the nature of population genetic data. Our framework relates key landscape genetics questions to

22 four levels of analysis: (i) Node-based methods, which model the spatial distribution of alleles at

23 sampling locations (nodes) from local site characteristics; these methods are suitable for

24 modeling adaptive genetic variation while accounting for the presence of spatial autocorrelation.

25 (ii) Link-based methods, which model the probability of gene flow between two patches (link)

26 and relate neutral molecular marker data to landscape heterogeneity; these methods are suitable

27 for modeling neutral genetic variation but are subject to inferential problems, which may be

28 alleviated by reducing links based on a network model of the population. (iii) Neighborhood-

29 based methods, which model the connectivity of a focal patch with all other patches in its local

30 neighborhood; these methods provide a link to metapopulation theory and landscape connectivity

31 modeling and may allow the integration of node- and link-based information, but applications in

32 landscape genetics are still limited. (iv) Boundary-based methods, which delineate genetically 
33 homogeneous populations and infer the location of genetic boundaries; these methods are suitable

34 for testing for barrier effects of landscape features in a hypothesis-testing framework. We

35 conclude that the power to detect the effect of landscape heterogeneity on the spatial distribution

36 of genetic variation can be increased by explicit consideration of underlying assumptions and

37 choice of an appropriate analytical approach depending on the research question.

38 Keywords: spatial statistics, neutral genetic variation, adaptive genetic variation, node analyses,

39 link analyses, neighborhood analyses, boundary analyses

40 


\section{Introduction}

43 Landscape genetics investigates neutral or adaptive genetic variation in spatially heterogeneous

44 landscapes (Holderegger and Wagner 2008; Storfer et al. 2007; Storfer et al. 2010). Landscape

45 genetic studies may have either $(i)$ an evolutionary focus aiming to infer the effects of micro-

46 evolutionary processes on genetic data while accounting for landscape effects such as isolation by

47 distance or matrix resistance (Storfer et al. 2010), or (ii) an ecological focus aiming to quantify

48 and test landscape effects on organism dispersal that potentially results in gene flow and spread

49 of adaptive genes (Manel et al. 2010; Spear et al. 2010). In the former case, spatial and landscape

50 effects are largely a nuisance for statistical hypothesis testing (Diniz-Filho et al. 2009; Legendre

51 and Legendre 1998), whereas in the latter case, the same effects may be the primary interest

52 (Fortin and Dale 2005). In both cases, landscape genetic studies are faced with conceptual and

53 statistical challenges related to inferring processes, which are not directly observable, from the

54 resulting spatial genetic structure (Anderson et al. 2010). For instance, the observed population

55 genetic structure represents a single realization of the underlying process, and independent

56 replication at the landscape scale is difficult to achieve (Wagner and Fortin 2005). The same

57 pattern may result, however, from different alternative processes, e.g., different dispersal

58 scenarios could explain the same population genetic structure or different series of historical

59 events can generate similar spatial patterns of genetic structure (Epperson 2003). Observed

60 genetic patterns are likely the result of several, potentially interacting processes, such as habitat

61 change (hereafter landscape dynamics), population dynamics, habitat selection, dispersal mode,

62 matrix resistance to dispersal, mating and reproduction system, mutation, selection and genetic 
63 drift (Fig. 1). Clearly, a single study is unlikely to address this full complexity but will identify

64 which processes to prioritize while making implicit or explicit assumptions on others.

65 In addition, methods for the spatial analysis of landscape heterogeneity (Fortin and Dale

66 2005; Wagner and Fortin 2005) and of population genetic structure (Epperson 2003; Slatkin and

67 Arter 1991; Sokal 1979) have largely been developed independently, drawing on different

68 theories and analytical methods, and thus lack a coherent framework for their joint analysis. In

69 order to apply and further develop methods for relating genetic data to landscape features,

70 landscape ecologists and spatial statisticians need to understand the specific nature of population

71 genetic data and population genetic methods, and population geneticists need to understand the

72 nature of landscape ecological data and spatial statistical methods.

73 Here, we stress how the choice of analysis method should be guided first by the research

74 question, then by the methods that can address the research question, and finally by the nature of

75 the data at hand. Consequently, this paper provides a framework for categorizing research

76 questions based on the processes considered (Fig. 1). We then classify statistical methods that

77 relate genetic data to landscape data according to four analysis levels that imply different data

78 models (Fig. 2). We thus aim to: (1) clarify where and how spatial and temporal processes matter

79 in landscape genetics, and (2) point out common misconceptions and prevent misunderstandings

80 of the complexity of issues that arise when moving from one field to another or in

81 interdisciplinary research collaborations between population geneticists, landscape ecologists,

82 spatial statisticians and other experts from related fields (Balkenhol et al. 2009a). 


\section{Processes to consider}

84 Most ecological and evolutionary processes are inherently spatially dynamic. Therefore,

85 landscape genetic studies need to consider the effects of processes both in space and time (Fig. 86 1).

Landscape change occurs due to various processes that create different types of spatial

88 dynamics: (i) Natural or anthropogenic disturbance may temporarily or permanently alter the

89 amount, quality and connectivity of habitat. Ecosystems and their organisms may be adapted to

90 natural disturbances, such as wildfires or insect outbreaks, and many natural disturbance regimes

91 result in a shifting mosaic with dynamic equilibrium at a larger spatial scale (Folke et al. 2004;

92 Holling 1992). However, anthropogenic disturbances may interact with natural disturbances in

93 complex ways, altering ecological system dynamics. (ii) Succession, e.g. triggered by a

94 disturbance event, changes species composition and species spatial distribution over time. (iii)

95 Climate change will gradually alter habitat quality, though extreme events such as drought may

96 trigger abrupt changes. (iv) Land-use change typically occurs as a discrete change from one land-

97 use type to another, though changes in land-use intensity such as fertilizer application may be 98 more gradual.

99 Similarly, key micro-evolutionary processes contribute to genetic diversity at neutral

100 loci or adaptive genes (Fig. 1). Even in a homogeneous environment, mutation will over time

101 change genetic variation, genetic drift will reduce genetic diversity due to the stochastic loss of

102 alleles from one generation to the next, and restricted dispersal can create spatial genetic structure

103 (isolation by distance, IBD; Lande 1991; Wright 1943; Wright 1948). Other micro-evolutionary

104 processes are directly affected by landscape spatial heterogeneity: environmental selection

105 depends on local habitat quality (Garant et al. 2007), whereas dispersal (movement from natal 
106 site to new site) and resulting migration (dispersal to new site followed by reproduction) may

107 depend on characteristics of the natal or new site as well as the physical distance between sites

108 (IBD) and the nature of the intervening landscape (matrix resistance), including the presence of

109 complete barriers to movement or more gradual differences in traversability or mortality (Spear et

110 al. 2010).

111 In ecological and evolutionary processes, both spatial and temporal constraints need to

112 be considered (Fig. 1). For instance, the study of selection in spatially heterogeneous landscapes

113 may be compromised by spatial autocorrelation introduced by restricted dispersal due to isolation

114 by distance or matrix resistance (Epperson 2003; Landguth et al. 2010). The fact that genetic

115 processes operate within time units of generations may introduce important time lags in the

116 response of spatial genetic structure to landscape change. Such time lags, referred to as ghost

117 landscape effects (Anderson et al. 2010), need to be differentiated from the contemporary

118 landscape effects. Dyer et al. (2010) showed that removing the effect of known phylogeographic

119 history may significantly improve our ability to assess landscape effects. Our conceptual

120 framework (Fig. 1) may help researchers identify the main processes of interest for their study,

121 those processes that are accounted for in the study design and statistical analysis, and any

122 processes that are not studied and for which the underlying assumptions should be made explicit.

123 For instance, a specific study focusing on landscape resistance to dispersal and gene flow would

124 explicitly investigate the processes of gene flow and landscape resistance while accounting for

125 IBD in the statistical analysis. It may ignore landscape change and thus assume that landscape

126 has been constant over many generations. It may further ignore evolutionary processes of

127 selection, mutation and drift, with underlying assumptions that the molecular markers studied are 
128 neutral and not linked to genes under selection, mutation is negligible at the temporal scale

129 studied, and local populations have not undergone recent bottlenecks.

\section{Research questions}

131 Landscape genetic studies may address a wide range of hypotheses and research questions,

132 depending on the focal micro-evolutionary process (gene flow vs. selection), the ecological or

133 evolutionary perspective (Storfer et al. 2010), and whether the intent is to study equilibrium

134 conditions or transient dynamics after landscape change (Table 1). Studies of gene flow

135 commonly assume that the molecular markers used are not affected by selection (Holderegger

136 and Wagner 2008). Studies of selection may rely on known adaptive genes (e.g., in model

137 organisms) or the identification of outlier loci as putatively adaptive loci or assumedly neutral

138 markers that may be linked to unknown adaptive genes due to proximity on the genome

139 (Holderegger and Wagner 2008; Holderegger et al. 2010).

140 The assumption that the amount, quality and connectivity of habitatremained constant

141 for a period long enough to reach an equilibrium between mutation, drift and migration may

142 rarely be met in real landscapes. Violations of underlying assumptions (such as Hardy-Weinberg

143 equilibrium) may compromise the validity of interpretation and generalization. The problem thus

144 is how to assess and account for the potential effect of such violations on the results. Other

145 studies explicitly focus on changes in dispersal, gene flow or adaptation in response to a known

146 landscape change or manipulation of amount, quality or connectivity of habitat, such as the

147 adding or removal of a putative barrier or the loss or addition of corridors and stepping stone

148 habitat. In such cases, it is important to measure contemporary gene flow that reflects current 
connectivity, which may be contrasted with estimates of gene flow prior to the landscape change

150 (Anderson et al. 2010).

151 In landscape genetics, it is often difficult to tease apart ecological and evolutionary

152 perspectives. The distinction is relevant, though, as misunderstandings may arise if one

153 researcher implicitly takes an ecological perspective, another evolutionary perspective, on what

154 may appear to be the same issue. When studying gene flow, an ecologist may want to use genetic

155 data as a proxy to infer dispersal rates among habitat patches with the goal of testing hypotheses

156 on matrix resistance that ultimately may lead to effective planning of habitat networks (Méndez

157 et al. 2011). Such studies often implicitly assume that all dispersal events will (or are equally

158 likely to) result in gene flow, but few studies explicitly investigate this discrepancy (Greenwald

159 2010).

$160 \quad$ From an evolutionary perspective, we are directly interested in inferring gene flow as a

161 measure of genetic connectivity, which may lead to the identification of populations at risk of

162 loss of viability and ultimately extinction. Small isolated populations are likely to experience

163 higher levels of inbreeding or Allee effects, which may result in depression of population fitness,

164 and reduction of genetic diversity through drift, which may reduce the potential for evolutionary

165 adaptation (Frankham 2005; Spielman et al. 2004). Such assessment of genetic connectivity often

166 assumes that new alleles arise in a population by immigration, not by mutation. Landscape

167 genetics however typically relies on markers with relatively high mutation rates, and estimates of

168 mutation rates for specific marker types carry high levels of uncertainty. The assumption that

169 mutation may be ignored at the population level may not hold, as illustrated by a recent study that

170 found a strong signal of mutation for both symbionts (fungus and alga) within replicate

171 populations of the lichen Lobaria pulmonaria (DalGrande et al. in press). 
Currently, most landscape genetic studies of adaptive variation take an ecological

173 perspective (Manel et al. 2010), such as aiming to identify outlier loci. A major challenge in this

174 context is accounting for spatial autocorrelation due to restricted dispersal or landscape spatial

175 heterogeneity. An evolutionary perspective may focus on the cohesion of spatially structured

176 populations in the context of speciation (typically assuming static landscape) or the spatial

177 dynamics of rapid evolutionary change in a changing environment (Holderegger and Wagner

178 2008). In the latter case, assumptions may be needed regarding the probability that the same

179 beneficial mutation has multiple independent origins. Note that the time lag in the genetic signal

180 is related to the rate of landscape change as compared to generation time.

\section{Approaches linking genetic and landscape data in landscape genetics}

182 Our framework is based on four analysis levels for relating neutral or adaptive genetic data to

183 landscape pattern (Fig. 2), depending on the research questions. These analysis levels can be

184 characterized by adopting terminology from graph theory, where nodes refer to habitat patches

185 and links to lines that connect any two patches (Dale and Fortin 2010). The four analysis levels

186 imply different data models, and in some cases it may be appropriate to convert data from one

187 data model to another to best match the analysis to the research question.

Node-based methods (Fig. 2A) relate the presence of adaptive genes or the genetic

189 diversity of local populations to environmental site conditions at sampling locations or to patch

190 attributes such as area or age. They thus address the question of what determines the presence and

191 abundance of alleles, or the genetic diversity, at a spatial location. Note that the question relating

192 to the presence and abundance of alleles is only meaningful for adaptive genetic variation, i.e.,

193 genes under selection or loci linked to such genes. 
At the node level, the analysis of allele frequency data from $n$ individuals, or predefined

195 local populations (e.g., demes, home ranges, nodes), is typically based on a response matrix Y

196 with $n$ rows and $m$ columns representing alleles. The explanatory matrix $\mathbf{X}$ with $n$ rows and $p$

197 columns represents the environmental and landscape predictors. Multivariate statistical methods

198 such as ordination (e.g., redundancy analysis RDA, canonical correspondence analysis CCA;

199 Legendre and Legendre 1998) can be used to predict the frequency of each allele $y$ from local site

200 conditions $x$. These methods can be used to identify outlier loci that are empirically associated

201 with landscape predictors. IBD may create spatial autocorrelation in the residuals, thus violating

202 the assumption of independent error of non-spatial linear models. If statistical tests (e.g., Moran's

203 I) indicate autocorrelated residuals, the analysis should be modified to account for spatial

204 autocorrelation due to IBD (Dray et al. in press; Wagner and Fortin 2005). This may be

205 accomplished by including an additional set of predictors $\mathbf{W}$ that model spatial structure in the

206 data at multiple scales based on the spatial locations of sampling points. The matrix $\mathbf{W}$ can be

207 used to partial out spatial variation by including it in the linear model as $\mathbf{Y} \sim \mathbf{X}+\mathbf{W}$. Methods for

208 creating matrix W has been developed as Principal Components of Neighborhood Matrices

209 (PCNM; Borcard et al. 2011) and generalized to Moran's Eigenvector Map (MEM; Dray et al.

210 2006) analysis. We can thus partial out all spatial variation when assessing the response of allele

211 frequencies Y to local site conditions X (Manel et al. 2010). However, this blanket approach may

212 also eliminate spatial variation unrelated to gene flow (Lichstein et al. 2002; Wagner and Fortin

213 2005). As an alternative, spatial regression could be used to explicitly build isolation by distance

214 into the model (Diniz-Filho et al. 2009).

215 Link-based methods (Fig. 2B) relate pair-wise genetic distance between individuals or

216 demes to their landscape distance (e.g., geographic distance, cost distance, presence or number of 
barriers) hypothesized to be related to the probability of dispersal and migration. They thus

218 address the question of how likely gene flow is between two patches. This analysis level may be

219 particularly relevant for assessing genetic connectivity, testing hypotheses on landscape

220 resistance, and identifying corridors for conservation applications.

At the link level, the analysis of allele frequency data from $n$ individuals, or demes, is

222 typically based on a $n \times n$ matrix $\mathbf{D}_{\mathbf{Y}}$ of pairwise genetic distances, and a set of $p$ matrices $\mathbf{D}_{\mathbf{X p}}$ of

223 size $n \times n$. Each matrix $\mathbf{D}_{\mathbf{X} \mathbf{p}}$ provides a measure of landscape distance $d_{i j}$ between two sampling

224 locations $i$ and $j$. In the simplest case, $d_{i j}$ is equal to the geographic distance, representing

225 isolation by distance. The effect of a barrier (e.g., mountain, river, road) can be represented with

226 a binary matrix. Similarly, the intervening landscape can be described in terms of the presence

227 (binary), absolute distance (e.g., in km) or relative distance (in percent of total distance) of each

228 cover type $p$ that an organism would have to traverse along a transect between sampling locations

$229 i$ and $j$ (e.g., Angelone et al. 2011). Alternatively, $d_{i j}$ may represent a potential cumulative cost for

230 an organism to traverse the intervening landscape between sampling locations $i$ and $j$. Cost values

231 of different cover types may be derived from field data on organism movement behavior, from

232 expert opinion, or through optimization given the genetic data (Spear et al. 2010). Cumulative

233 cost $d_{i j}$ is commonly calculated either $(i)$ along the shortest physical distance (transect) between

234 locations $i$ and $j$, (ii) along a corridor of specified width, (iii) along the least-cost path, or (iv) as

235 total resistance (McRae et al. 2008) integrating over all possible paths (Spear et al. 2010).

236 Mantel tests have been used to test the association between two distance matrices $\mathbf{D}_{\mathbf{Y}}$

237 and $\mathbf{D}_{\mathbf{X} 1}$, and partial Mantel tests allow accounting for a third matrix $\mathbf{D}_{\mathbf{X} 2}$. These methods

238 evaluate the (linear or rank) correlation between vectors of pairwise distances $d_{i j}$. Appropriate

239 permutation tests need to be used for significance testing to account for the inflated sample size, 
240 as each vector has length $n(n-1) / 2$ (Legendre and Fortin 2010). (Partial) Mantel tests have been

241 shown to have low statistical power in general (Legendre and Fortin 2010), thus likely to fail to

242 detect an effect especially with small data sets. On the other hand, landscape genetic studies often

243 experience the opposite problem that many candidate models show statistically significant effects

244 and criteria are needed to identify the most important factors (Cushman and Landguth 2010;

245 Cushman et al. 2006). Multiple regression of distance matrices (Legendre and Legendre 1998;

246 Legendre and Fortin 2010) rephrases the problem from correlation to regression and allows

247 modeling of the response matrix $\mathbf{D}_{\mathbf{Y}}$ by a number of matrices $\mathbf{D}_{\mathbf{X} 1}, \mathbf{D}_{\mathbf{X} 2}, \ldots, \mathbf{D}_{\mathbf{X p}}$ simultaneously.

248 The power of link-based analysis may be improved by reducing the full distance matrix to a

249 subset of links, either based on generic spatial graph models (e.g., planar graph that connects only

250 adjacent sampling locations; Dale and Fortin 2010) or using population graphs based on

251 conditional genetic distance (Dyer et al. 2010; Garroway et al. 2011). More research is needed to

252 develop and test approaches at model selection, e.g., using criteria such as AIC or BIC (Ward

253 2008) taking into account the inflated sample size in regression of distance matrices as well as

254 inferential problems related to spatial autocorrelation in predictor and response variables, which

255 in turn affect the effective sample size.

256 Neighborhood-based methods (Fig. 2C) relate genetic diversity of demes, or their

257 genetic differentiation, to attributes of the local landscape context, either within a certain radius

258 around the sampling location or as a function of the neighboring patches, with weights inversely

259 proportional to their landscape distance scaled by the dispersal ability of the organism. They thus

260 address the question of how connected each patch is to all nearby patches, and how such

261 connectivity affects genetic diversity or differentiation at the level of demes (Keyghobadi et al.

262 2005). Such neighborhood-level analysis is compatible with a patch-based metapopulation 
263 perspective and often uses a patch connectivity model $S_{i}$ (Balkenhol et al. 2009b; Bulman et al.

264 2007; James et al. 2011) to quantify local landscape context in a node-based connectivity

265 measure. While link-based methods model connectivity between pairs of nodes, neighborhood-

266 level analysis integrates connectivity for a focal patch across all other patches in its

267 neighborhood. Here, a $n \times m$ matrix $\mathbf{Y}$ of allele frequencies, or a single vector $y$ of an aggregate

268 measure such of genetic diversity or differentiation, is related to a $n \times p$ matrix $\mathbf{S}$ of patch

269 connectivity measures, where each variable $S_{p}$ contains the connectivity value $S_{i}=\Sigma_{\mathrm{j}} \mathrm{X}_{\mathrm{pj}}$ for each

270 patch $i$ with respect to landscape predictor $p$. Each patch connectivity measure may be calculated

271 for a single environmental factor, or for a resistance surface that integrates multiple factors

272 (Balkenhol et al. 2009b; Foll and Gaggiotti 2006). Note that summarizing landscape features, e.g.

273 road density or the proportions of different habitat types, within a given radius around each

274 sampling location, irrespective of the other sampling locations, does not provide connectivity

275 measures $\mathbf{S}$ but node-based variables $\mathbf{X}$ that quantify the local landscape context.

276 Technically, this type of analysis brings information about the pairwise links back into a

277 node-based data structure. This means that matrix S, containing variables modeling dispersal

278 from neighboring patches (potentially affecting gene flow), may be combined with a matrix $\mathbf{X}$

279 that contains additional variables relating to local site conditions (potentially affecting selection)

280 in the same analytical framework. It is important to keep in mind what exactly is being modeled.

281 In the multivariate case, allele frequencies in matrix $\mathbf{Y}$ are predicted by patch connectivity. For

282 instance, this could mean that we predict the presence of an allele from an overall patch

283 connectivity measure or from the amount of woodland cover in the local landscape around the

284 patch. Although conceptually questionable for neutral markers, simulation results indicate that

285 such neighborhood-based methods may perform comparably well under a range of landscape 
genetic scenarios (Balkenhol et al. 2009b). More research is needed to clarify the applicability of the approach for different research questions, including the application of distance-based RDA where $\mathbf{Y}$ is replaced by a genetic distance matrix $\mathbf{D}_{\mathbf{y}}$. Gravity models provide a promising avenue for the joint modeling of node- and link-related processes (Murphy et al. 2010).

Boundary-based methods (Fig. 2D), which include boundary detection algorithms and spatial Bayesian clustering methods, aim to delineate discrete or admixed populations in space

292 (Barbujani et al. 1989; Safner et al. 2011). The determination of whether individual genetic data

293 (Y) collected at different locations stem from a panmictic population or from several

294 geographically and genetically distinct populations is an important goal in conservation genetics

295 (Frankham 2009). When spatially distinct populations are identified based on genetic data, the 296 next step in boundary-level methods consists in determining which landscape features separate

297 them. Boundary-based methods thus address the question of what landscape features constitute a 298 barrier to gene flow.

Spatially distinct populations can be delineated using boundary detection methods such

300 as Monmonier's algorithm (Monmonier 1973) or Womble's bilinear algorithm (Barbujani et al.

301 1989). In essence, using quantitative data (e.g., gene frequencies, environmental data) these

302 algorithms identify boundaries between adjacent locations (based on Delaunay links) as highest

303 rates of change $\beta$. Hence for each link $d_{\mathrm{ij}}$ between directly adjacent sampling locations $i$ and $j$

304 (nodes or grid cells), a rate of change value $\beta_{Y}$ indicates how likely the link is to cross a genetic

305 boundary. This can be reduced to a binary classification of links as boundary or non-boundary,

306 either based on significance testing or an arbitrary threshold value. Similarly, boundaries in

307 environmental conditions can be identified by assessing spatial rate of change $\beta_{\mathrm{X}}$. 
309 result in a categorical vector $y$ of length $n$ that specifies for each observation (individual or deme)

310 to which of $k$ discrete genetic populations it has been assigned. If the method allows admixture,

311 the response is a $n \times k$ matrix $\mathbf{M}$ with probabilities of membership of each of $n$ observations for

312 each of $k$ genetic populations, with row sums adding to 1 . The presence of isolation by distance

313 may lead to spurious boundary detection (Safner et al. 2011). Spatial Bayesian clustering as

314 proposed by François and Durand (2010) adds a spatial graph constraint to determine spatial

315 cluster memberships.

316 Once genetic boundaries, or spatial cluster memberships, are identified, the next step is

317 to relate them to environmental or landscape features. This can be achieved by testing the degree

318 of spatial overlap (Jacquez et al. 2000; Oden et al. 1993) between genetic boundaries and

319 landscape features $(\mathbf{X})$ hypothesized to restrict dispersal. The spatial coincidence between the

320 genetic $\left(\beta_{\mathrm{Y}}\right)$ and landscape boundaries $\left(\beta_{\mathrm{X}}\right)$ can be assessed using boundary overlap statistics

321 (Barbujani and Sokal 1991; Fortin et al. 1996; Oden et al. 1993; St-Louis et al. 2004) to test

322 specific hypotheses about landscape effects on the genetic data. Alternatively, the relationship

323 between the cluster memberships and environmental conditions $\mathbf{X}$ can be tested using ancestry

324 distribution models as implemented in POPS software (Prediction of Population genetic

325 Structure; Durand et al. 2009; Jay 2011).

\section{Conclusions}

327 Landscape genetics research questions require analysis at different spatio-temporal scales and 328 thus different data models, analysis levels and statistical methods. Node-based methods are the 329 natural choice for studying selection, link-based methods for studying gene flow. The barrier 
330 effect of new landscape features may best be detected by testing the overlap between genetic

331 boundaries in contemporary gene flow assessed before and after establishment of the

332 hypothesized barrier. Novel questions relating to the spread of adaptive variation in changing

333 landscapes will require methods that integrate node- and link-based analysis, for which

334 neighbourhood-level analysis may be best suited. Statistically sound and powerful methods are

335 needed for each analysis level, as questions are not easily transferable to a different analysis

336 level.

337 The presence of multiple processes, and the absence of equilibrium conditions, may

338 compromise many methods. Landscape genetics needs a true integration of population genetic

339 and ecological analysis methods and the development of new methods that take into account the

340 complexity of landscape genetic data.

\section{Acknowledgments}

342 This work resulted from a Distributed Graduate Seminar (Developing Best Practices for Testing

343 Landscape Effects on Gene Flow), conducted through the National Center for Ecological

344 Analysis and Synthesis, a center funded by the National Science Foundation grant \#EF-0553768,

345 the University of California, Santa Barbara, and the State of California. It was also supported by

346 NSERC discovery grants to HHW and MJF. We thank Michelle DiLeo and Yessica Rico for their

347 thoughtful comments.

349 References

350 Anderson CD, Epperson BK, Fortin MJ, Holderegger R, James PMA, Rosenber MS, Sribner KT, gene flow. Mol Ecol 19:3565-3575 
353

354

355

356

357

358

359

360

361

362

363

364

365

366

367

368

369

370

371

372

373

374

375

376

377

378

379

380

381

382

383

384

385

386

387

388

389

390

391

392

393

394

395

396

397

Angelone S, Kienast F, Holderegger R (2011) Where movement happens: scale-dependent landscape effects on genetic differentiation in the European tree frog. Ecography 34:714722

Balkenhol N, Gugerli F, Cushman SA, Waits L, Coulon A, Arntzen J, Holderegger R, Wagner $\mathrm{HH}$ (2009a) Identifying future research needs in landscape genetics: where to from here? Landscape Ecol 24:455-463

Balkenhol N, Waits LP, Dezzani RJ (2009b) Statistical approaches in landscape genetics: an evaluation of methods for linking landscape and genetic data. Ecography 32:818-830

Barbujani G, Sokal RR (1991) Genetic population structure of Italy. II. Physical and cultural barriers to gene flow. Am J Hum Genet 48:398-411

Barbujani G, Oden NL, Sokal RR (1989) Detecting regions of abrupt change in maps of biological variables. Syst Zool 38:376-389

Borcard D, Gillet F, Legendre P (2011) Numerical ecology with R. Springer, New York

Bulman CR, Wilson RJ, Alison R. Holt, Bravo LG, Early RI, Warren MS, Thomas CD (2007) Minimum viable metapopulation size, extinction debt, and the conservation of a declining species. Ecol Appl 17:1460-1473

Cushman SA, Landguth EL (2010) Spurious correlations and inference in landscape genetics. Mol Ecol 19:3592-3602

Cushman S, McKelvey K, Hayden J, Schwartz M (2006) Gene flow in complex landscapes: testing multiple hypotheses with causal modeling. Am Nat 168:486-499

Dale MRT, Fortin MJ (2010) From graphs to spatial graphs. Annual Review of Ecology, Evolution, and Systematics 41:21-38

DalGrande F, Widmer I, Wagner HH, Scheidegger C (2012) Vertical and horizontal photobiont transmission within populations of a lichen symbiosis. Mol Ecol. doi: 10.1111/j.1365294X.2012.05482.X

Dawson KJ, Belkhir K (2001) A Bayesian approach to the identification of panmictic populations and the assignment of individuals. Genet Res 78:59-77

Diniz-Filho JAF, Nabout JC, Telles MPdC, Soares TN, Rangel TFLVB (2009) A review of techniques for spatial modeling in geographical, conservation and landscape genetics. Genetics and molecular biology 32:203-211

Dray S, Pélissier R, Couteron P, Fortin MJ, Legendre P, Peres-Neto PR, Bellier E, Bivand R, Blanchet FG, De Cáceres M, Dufour A, Heegard E, Jombart T, Munoz F, Oksanen J, Thioulouse J, Wagner HH (in press) Community ecology in the age of multivariate multiscale spatial analysis. Ecological Monographs

Dray S, Legendre P, Peres-Neto PR (2006) Spatial modelling: a comprehensive framework for principal coordinate analysis of neighbour matrices (PCNM). Ecol Model 196:483-493

Durand E, Jay F, Gaggiotti OE, François O (2009) Spatial Inference of Admixture Proportions and Secondary Contact Zones. Mol Biol Evol 26:1963-1973

Dyer RJ, Nason JD, Garrick RC (2010) Landscape modelling of gene flow: improved power using conditional genetic distance derived from the topology of population networks. Mol Ecol 19:3746-3759

Epperson BK (2003) Geographical genetics. Princeton University Press, Princeton, NJ

Folke C, Carpenter S, Walker B, Scheffer M, Elmqvist T, Gunderson L, Holling CS (2004) Regime shifts, resilience, and biodiversity in ecosystem management. Annual Review of Ecology, Evolution and Systematics 35:557-581 
398

399

400

401

402

403

404

405

406

407

408

409

410

411

412

413

414

415

416

417

418

419

420

421

422

423

424

425

426

427

428

429

430

431

432

433

434

435

436

437

438

439

440

441

442

443

Foll M, Gaggiotti OE (2006) Identifying the environmental factors that determine the genetic structure of populations. Genetics 174:875-891

Fortin MJ, Dale MRT (2005) Spatial analysis: a guide for ecologists. Cambridge University Press, New York

Fortin MJ, Drapeau P, Jacquez GM (1996) Quantification of the spatial co-occurrences of ecological boundaries. Oikos 77:51-60

François O, Durand E (2010) Spatially explicit Bayesian clustering models in population genetics. Molecular Ecology Resources 10:773-784

Frankham R (2005) Genetics and extinction. Biol Conserv 126:131-140

Frankham R (2009) Introduction to conservation genetics. Cambridge University Press, New York

Garant D, Kruuk LEB, McCleery RH, Sheldon BC (2007) The effects of environmental heterogeneity on multivariate selection on reproductive traits in female great tits. Evolution 61:1546-1559

Garroway CJ, Bowman J, Wilson PJ (2011) Using a genetic network to parameterize a landscape resistance surface for fishers, Martes pennanti. Mol Ecol 20:3978-3988

Greenwald KR (2010) Genetic data in population viability analysis: case studies with ambystomatid salamanders. Animal Conservation 13:115-122

Holderegger R, Buehler D, Gugerli F, Manel S (2010) Landscape genetics of plants. Trends Plant Sci 15:675-683

Holderegger R, Wagner HH (2008) Landscape genetics. Bioscience 58:199-207

Holling CS (1992) Cross-scale morphology, geometry, and dynamics of ecosystems. Ecol Monogr 62:447-502

Jacquez GM, Maruca S, Fortin MJ (2000) From fields to objects: A review of geographic boundary analysis. Journal of geographical systems 2:221-241

James PMA, Coltman DW, Murray BW, Hamelin RC, Sperling FAH (2011) Spatial Genetic Structure of a Symbiotic Beetle-Fungal System: Toward Multi-Taxa Integrated Landscape Genetics. PLoS ONE 6:e25359

Jay F (2011) PoPS: Prediction of Population genetic Structure - Program documentation and tutorial. University Joseph Fourier, Grenoble, France

Keyghobadi N, Roland J, Matter SF, Strobeck C (2005) Among- and Within-Patch Components of Genetic Diversity Respond at Different Rates to Habitat Fragmentation: An Empirical Demonstration. Proceedings: Biological Sciences 272:553-560

Lande R (1991) Isolation by distance in a quantitative trait. Genetics 128:443-452

Landguth EL, Cushman S, Murphy MA, Luikart G (2010) Relationships between migration rates and landscape resistance assessed using individual-based simulations. Molecular Ecology

Resources 10:854-862

Legendre P, Fortin MJ (2010) Comparison of the Mantel test and alternative approaches for detecting complex multivariate relationships in the spatial analysis of genetic data.

Molecular Ecology Resources 10:831-844

Legendre P, Legendre L (1998) Numerical ecology. Elsevier, New York

Lichstein JW, Simons TR, Shriner SA, Franzreb KE (2002) Spatial autocorrelation and autoregressive models in ecology. Ecol Monogr 72:445-463

Manel S, Joost S, Epperson BK, Holderegger R, Storfer A, Rosenberg MS, Scribner KT, Bonin A, Fortin MJ (2010) Perspectives on the use of landscape genetics to detect genetic adaptive variation in the field. Mol Ecol 19:3760-3772 
McRae BH, Dickson BG, Keitt TH, Shah VB (2008) Using circuit theory to model connectivity in ecology, evolution, and conservation. Ecology 89:2712-2724

Méndez M, Tella JL, Godoy JA (2011) Restricted gene flow and genetic drift in recently fragmented populations of an endangered steppe bird. Biol Conserv 144:2615-2622

Monmonier MS (1973) Maximum-difference barriers: an alternative numerical regionalization method. Geographical Analysis 5:245-261

Murphy MA, Dezzani RJ, Pilliod DS, Storfer A (2010) Landscape genetics of high mountain frog metapopulations. Mol Ecol 19:3634-3649

Oden NL, Sokal R, Fortin MJ, Goebl H (1993) Categorical wombling: detecting regions of significant change in spatially located categorical variables. Geogr Anal 25:315-336

Safner T, Miller MP, McRae BH, Fortin MJ, Manel S (2011) Comparison of bayesian clustering and edge detection methods for inferring boundaries in landscape genetics. International journal of molecular sciences 12:865-889

Slatkin M, Arter HE (1991) Spatial autocorrelation methods in population genetics. Am Nat 138:499-517

Sokal RR (1979) Testing statistical significance of geographic variation patterns. Syst Zool 28:227-232

Spear SF, Balkenhol N, Fortin MJ, McRae BH, Scribner KT (2010) Use of resistance surfaces for landscape genetic studies: considerations for parameterization and analysis. Mol Ecol 19:3576-3591

Spielman D, Brook BW, Frankham R, Schaal BA (2004) Most Species Are Not Driven to Extinction before Genetic Factors Impact Them. Proc Natl Acad Sci USA 101:1526115264

St-Louis V, Fortin MJ, Desrochers A (2004) Spatial association between forest heterogeneity and breeding territory boundaries of two forest songbirds. Landscape Ecol 19:591-601

Storfer A, Murphy MA, Spear SF, Holderegger R, Waits LP (2010) Landscape genetics: where are we now? Mol Ecol 19:3496-3514

Storfer A, Murphy MA, Evans JS, Goldberg CS, Robinson S, Spear SF, Dezzani R, Delmelle E, Vierling L, Waits LP (2007) Putting the 'landscape' in landscape genetics. Heredity 98:128142

Wagner HH, Fortin MJ (2005) Spatial analysis of landscapes: concepts and statistics. Ecology 86:1975-1987

Ward EJ (2008) A review and comparison of four commonly used Bayesian and maximum likelihood model selection tools. Ecol Model 211:1-10

Wright S (1948) On the roles of directed and random changes in gene frequency in the genetics of populations. Evolution 2:279-294

Wright S (1943) Isolation by distance. Genetics 28:114-138 


\section{Tables}

Table 1. Examples of landscape genetics research questions and common assumptions.

\begin{tabular}{|c|c|c|c|}
\hline $\begin{array}{l}\text { Micro-evolutionary } \\
\text { Processes }\end{array}$ & Analysis context & $\begin{array}{l}\text { Static Landscape } \\
\text { Assumption: } \\
\text { mutation -drift- } \\
\text { migration } \\
\text { equilibrium }\end{array}$ & $\begin{array}{l}\text { Changing Landscape } \\
\text { Assumption: } \\
\text { Contemporary gene flow } \\
\text { is measured }\end{array}$ \\
\hline \multirow[t]{2}{*}{$\begin{array}{l}\text { Gene Flow } \\
\text { Assumption: } \\
\text { Markers are not } \\
\text { affected by selection }\end{array}$} & $\begin{array}{l}\text { Ecological: } \\
\text { Landscape } \\
\text { connectivity }\end{array}$ & $\begin{array}{l}\text { What is the } \\
\text { resistance of } \\
\text { different land-cover } \\
\text { types to the dispersal } \\
\text { of a species of } \\
\text { interest? }\end{array}$ & $\begin{array}{l}\text { How does a new barrier } \\
\text { affect the dispersal of a } \\
\text { species of concern? }\end{array}$ \\
\hline & $\begin{array}{l}\text { Evolutionary: } \\
\text { Genetic connectivity }\end{array}$ & $\begin{array}{l}\text { Is there enough gene } \\
\text { flow to maintain a } \\
\text { viable population? }\end{array}$ & $\begin{array}{l}\text { Does a new barrier } \\
\text { disrupt gene flow for a } \\
\text { species of concern? }\end{array}$ \\
\hline \multirow[t]{2}{*}{$\begin{array}{l}\text { Selection } \\
\text { Assumption: Loci } \\
\text { are under selection } \\
\text { or linked to adaptive } \\
\text { genes }\end{array}$} & $\begin{array}{l}\text { Ecological: } \\
\text { Adaptation to } \\
\text { selective } \\
\text { environment } \\
\end{array}$ & $\begin{array}{l}\text { Which loci are } \\
\text { potentially linked } \\
\text { with adaptive genes? }\end{array}$ & $\begin{array}{l}\text { Does a change in habitat } \\
\text { quality trigger rapid } \\
\text { evolutionary change? }\end{array}$ \\
\hline & $\begin{array}{l}\text { Evolutionary: } \\
\text { Cohesion of a } \\
\text { spatially structured } \\
\text { population }\end{array}$ & $\begin{array}{l}\text { How does gene flow } \\
\text { affect the frequency } \\
\text { of adaptable genes? }\end{array}$ & $\begin{array}{l}\text { How do beneficial } \\
\text { mutations spread across a } \\
\text { spatially structured } \\
\text { population? }\end{array}$ \\
\hline
\end{tabular}


489

490

491

492

493

\begin{tabular}{|c|c|c|}
\hline & Spatial pattern description of $\mathbf{Y}$ & Spatial modeling of $\mathbf{Y}$ \\
\hline Node & $\begin{array}{l}\text { Global spatial autocorrelation } \\
\text { (Moran's I, Geary's } c \text {, variography) } \\
\text { Spatial interpolation (Kriging) } \\
\text { sPCA }\end{array}$ & $\begin{array}{l}\text { Spatial regression } \\
\text { Gravity model } \\
\text { Partial direct ordination using MEM }\end{array}$ \\
\hline Link & Mantel correlogram & $\begin{array}{l}\text { Partial Mantel tests } \\
\text { Partial regression of distance matrices }\end{array}$ \\
\hline Neighborhood & $\begin{array}{l}\text { Local spatial autocorrelation } \\
\text { (Moran's } I, \text { Geary's } c \text { ) }\end{array}$ & Geographical weighted regression \\
\hline Boundary & $\begin{array}{l}\text { Boundary detection (Wombling, } \\
\text { Monmonier) } \\
\text { Spatial Bayesian clustering (TESS) }\end{array}$ & $\begin{array}{l}\text { Correlation between boundaries } \\
\text { (Boundary overlap statistics) or clusters } \\
\text { with environmental/landscape features } \\
\text { (POPS) }\end{array}$ \\
\hline
\end{tabular}

Table 2: Examples of spatial analysis methods that can be used to describe or model spatial patterns in one or more response variables $\mathbf{Y}$ at each of the four analysis levels. 


\section{Figure captions}

Fig. 1. Conceptual model of how space and time affect relevant processes in the fields of

500 landscape ecology (left box) and population genetics (right box). The primary focus of landscape

501 genetics is how landscape spatial and temporal heterogeneity shape the spatial distribution of

502 genetic variation by modifying environmental selection and gene flow (grey box). By linking

503 concepts from landscape ecology and population genetics, landscape genetics embraces both

504 spatial and temporal dimensions and needs to consider the relevant ecological and evolutionary

505 processes, either explicitly or through assumptions.

Fig. 2. Four analysis levels at which landscape genetic data can be analyzed: (A) At the

508 node level, the alleles ( $\mathbf{Y})$ at each sampling location $a, b$ or $c$ are related to environmental

509 conditions or landscape features $(\mathbf{X})$ observed at the same location. Alternatively, Y may

510 represent an aggregate measure of genetic diversity such as allelic richness. Nodes of different

511 sizes refer either to different genetic values or habitat patch sizes. (B) At the link level, genetic

512 distance $\mathbf{D}_{\mathrm{Y}}$ between pairs of sampling locations $a b, a c$ and $b c$ is related to distance-based

513 landscape data $\mathbf{D}_{\mathrm{X}}$ describing the intervening matrix along each link. (C) At the neighborhood

514 level, the alleles $\mathbf{Y}$ (or diversity measure, see above) at sampling location $a$ are related to a patch-

515 level connectivity measure $\Sigma_{\mathrm{j}} \mathrm{X}_{\mathrm{j}}$ that quantifies local landscape context (see text). (D) At the

516 boundary level, spatially contiguous, discrete populations $a, b$ and $c$ are inferred from genetic

517 data and overlaid with landscape features to identify barriers to gene flow. This may be achieved

518 by relating spatial rates of change in genetic data, $\beta_{Y}$, to spatial rates of change in landscape 
519 predictors $\beta_{\mathrm{X}}$. Alternatively, $\beta$ may denote between-cluster components of variance in $\mathbf{X}$ or $\mathbf{Y}$ 520 (see text).

521

522 
523 Figures

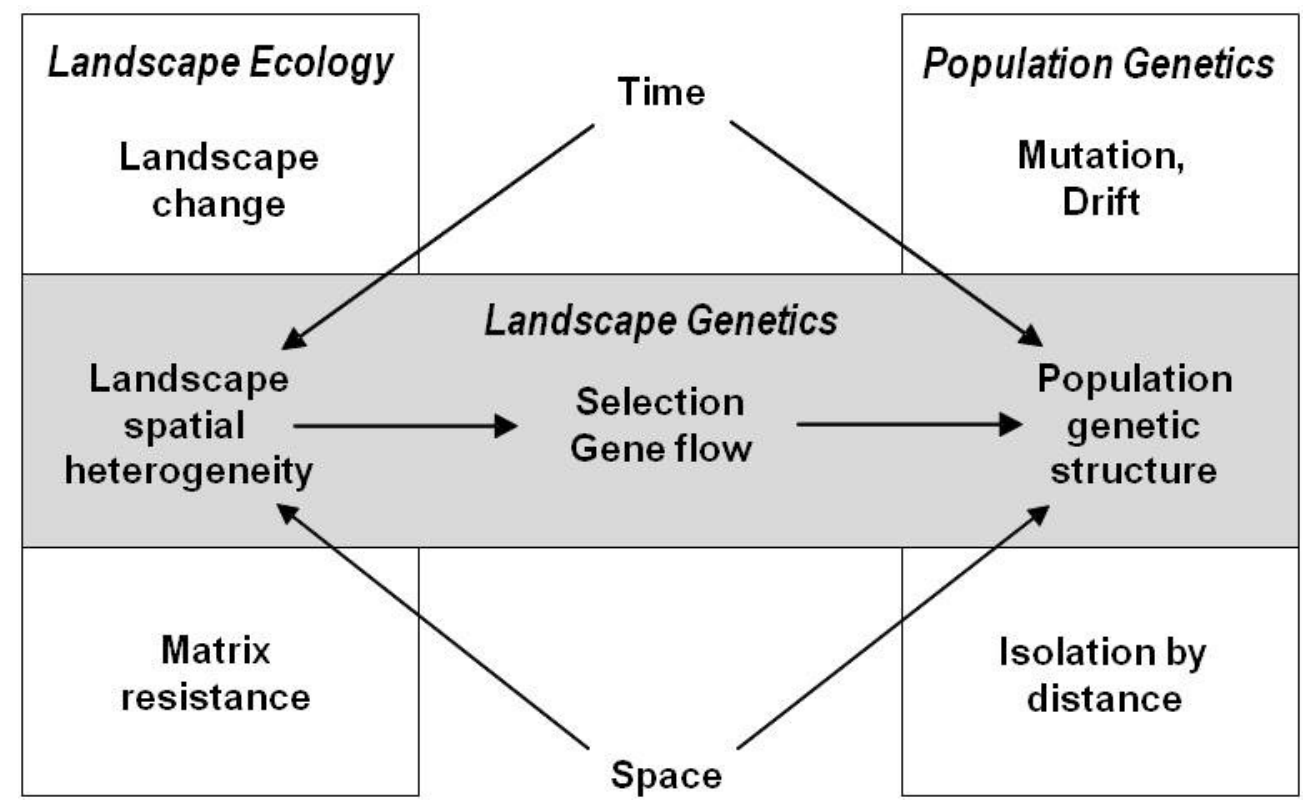

Fig. 1 
A: Node level

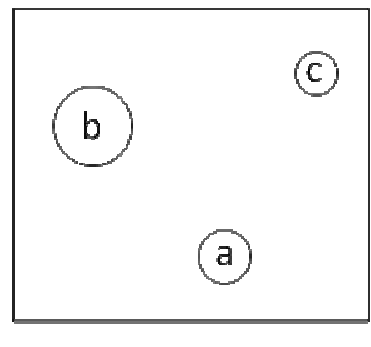

$Y \sim X$
B: Link Ievel

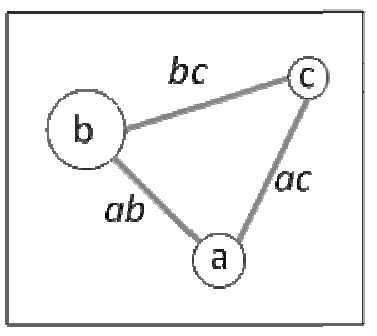

$\mathrm{D}_{\mathrm{Y}} \approx \mathrm{D}_{\mathrm{X}}$
C: Neighborhood level

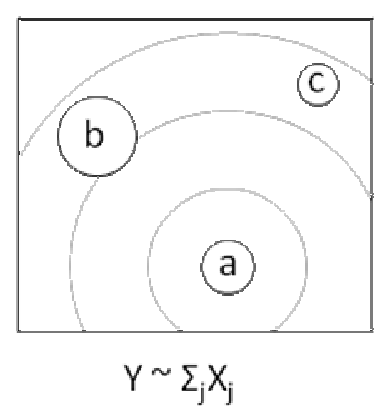

D: Boundary level

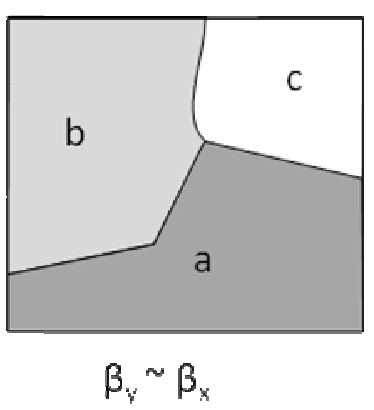

$531 \quad$ Fig. 2 